\title{
Pseudotumoral Demyelinating Lesions: A Presentation of Acute Disseminated Encephalomyelitis
}

\author{
Rachid Belfkih Omar Ghomari Khayat Hind H'daidane \\ Fatima Zahra El Amrani
}

Neurology Department, University Hospital Center of Tangier-Tetouan-Alhoceima, Tangier, Morocco

\section{Keywords}

Case report - Pseudotumoral demyelinating lesions $\cdot$ Multiple sclerosis $\cdot$ Acute disseminated encephalomyelitis

\begin{abstract}
Pseudotumoral forms of demyelination are related to central nervous system demyelinating disorders, usually considered to be an atypical presentation of multiple sclerosis including its different varieties such as Balo's, Schilder's, and Marburg diseases. These lesions could also be seen in myelin oligodendrocyte glycoprotein antibody-associated demyelination, acute disseminated encephalomyelitis (ADEM), and neuromyelitis optica spectrum disorder. The pseudotumoral aspect may be mistakenly considered as an abscess or a cancerous tumor, in which case, patients could endure unnecessary possibly harmful brain biopsy and have a delay in their disease diagnostics and management. Once latter differential diagnosis is discarded, pseudotumoral demyelination prompts uncertainties concerning the nature of the underlying demyelinating condition as prognosis and management differ from multiple sclerosis to other syndromes, especially whether a chronic treatment is needed or not. In this case report, we present a 35-year-old male patient hospitalized in the department of neurology for a rapidly progressive onset of encephalopathy and polyfocal neurological deficits, with pseudotumoral lesions shown on brain MRI. On further investigations, ADEM was the more likely diagnosis that could fit the patient's clinical and radiological presentation. Thence, he was put on high dose of intravenous corticosteroids, with a followed good recovery within the first week of the treatment.
\end{abstract}


Belfkih et al.: Pseudotumoral Presentation of Acute Disseminated Encephalomyelitis

\section{Introduction}

Pseudotumoral lesions are an atypical presentation of demyelinating disorders, commonly associated with multiple sclerosis of different varieties and occasionally linked to other demyelinating processes, such as myelin oligodendrocyte glycoprotein (MOG) antibodyassociated demyelination, acute disseminated encephalomyelitis (ADEM), and neuromyelitis optica spectrum disorder (NMOSD) [1]. Their frequencies are still uncertain, with an estimated prevalence of 3 cases per million per inhabitants [2] and an occurrence of $1.4-8.2 \%$ in multiple sclerosis patients [1]. Herein, we present a case of a male adult with a history of rapidly progressive onset of encephalopathy and polyfocal neurological signs, and whose brain MRI has exhibited pseudotumoral lesions. Further evaluation ensued with the diagnosis of ADEM.

\section{Clinical Presentation}

A 35-year-old male patient presented to the department of neurology with encephalopathy and neurological deficits. His family reported that over the course of the last month, he started suffering from asthenia, anorexia, vomiting, and mild headaches. His speech became slurred, and he started having behavioral disorders, such as confusion, anxiety, and insomnia. Over the period of 8 days before admission, he developed right hemiparesis, facial asymmetry, and aphasia. Prior to the onset of his condition, he has not submitted any complaints of blurred vision or increased sensitivity to light to his surroundings. The patient has a past medical history of cannabis use and a urinary tract infection that goes back to 15 days before the first symptom onset. He has no significant family history to report.

On physical examination, vital parameters such as the pulse, respiratory rate, blood pressure, and temperature were within standard values. On neurological examination, the patient was agitated. He had right hemiplegic gait and normal posture with no ataxia, a motor deficit in his right lower and upper limbs with a muscle strength of 1/5 on the Medical Research Council (MRC) scale, the left side was normal, and there was no sensory deficit. Signs of meningeal irritation were absent. At the cranial nerve examination, the patient had a central facial palsy on the right side of his face. Deep tendon reflexes on the right side were brisk and hyperactive with indifferent plantar reflex. There was no tremor or other involuntary movements, with intact coordination. The rest of the physical examination revealed no abnormalities.

Investigations included a complete blood count that revealed a high white blood count at $20.10^{3} / \mathrm{mm}^{3}$ and a thrombopenia of $7.10^{3} / \mathrm{mm}^{3}$, while the C-reactive protein test and sedimentation rate were normal. Serological tests for HIV and syphilis were negative. Anti-myelin oligodendrocyte glycoprotein antibodies were not found. On funduscopic examination, there was no evidence of papilledema. A spinal tap was performed, and cerebrospinal fluid (CSF) analysis showed a normal cell count and protein levels, with negative viral and bacterial cultures. Oligoclonal band testing in CSF was negative. The brain computerized tomography scan disclosed large expansive hypodense lesions in both the hemispheres, with ring-shaped enhancement in post-contrast images. Brain magnetic resonance imaging showed in T2-weighted and FLAIR sequences multiple large, hyperintense, and tumefactive demyelinating lesions, with poorly defined borders and minimal surrounding edema, involving bilateral central white matter (WM), juxtacortical WM, periventricular area, and basal ganglia. Some lesions are multilayered consisting with Balo's concentric sclerosis (shown in Fig. 1). On T1-weighted images, pre-contrast sequences show large hypointense lesions taking contrast in post-gadolinium injection sequences at the periphery demonstrating an open ring-shaped

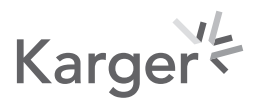



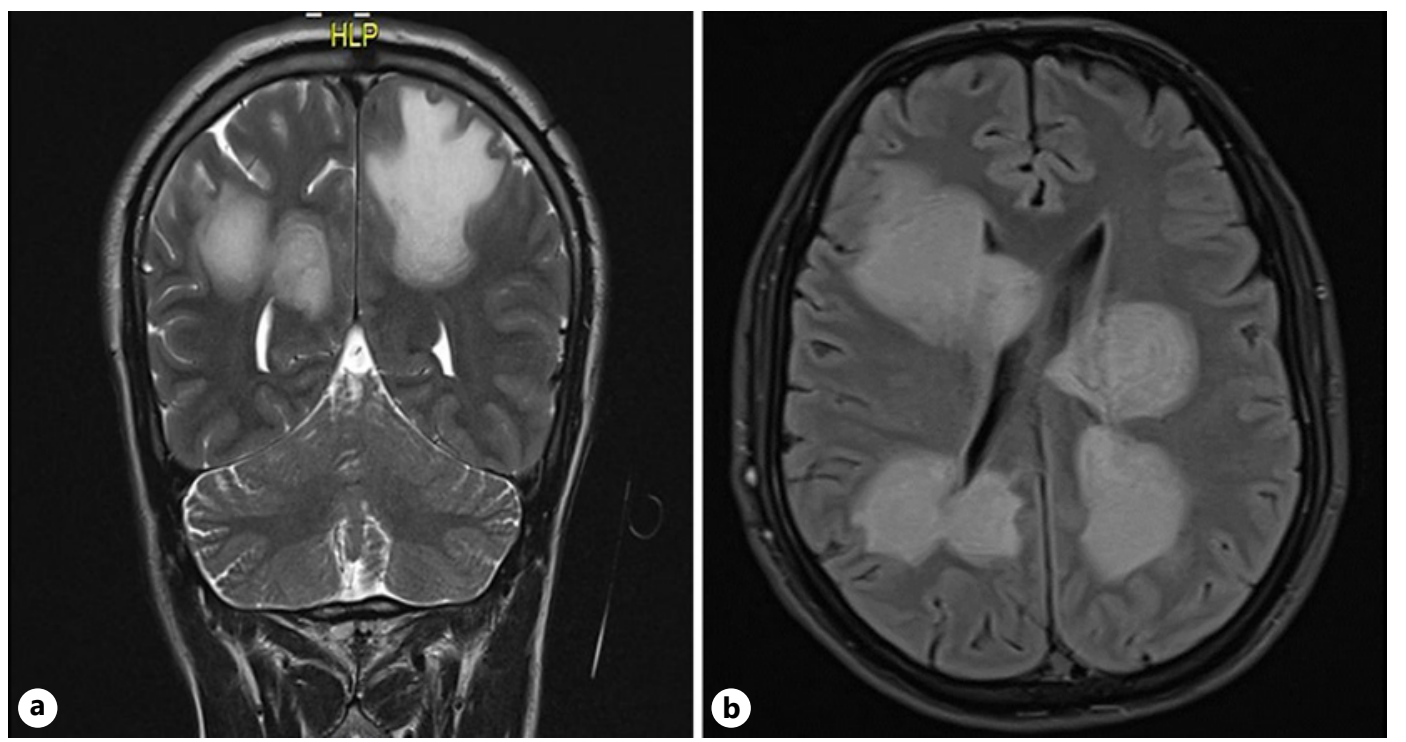

Fig. 1. T2-weighted (a) and FLAIR MRI (b) sequences showing multiple large, hyperintense and tumefactive demyelinating lesions, with poorly defined borders and minimal surrounding edema, involving bilateral central WM, the juxtacortical WM, periventricular area, and basal ganglia. Some lesions are multilayered consisting with Balo's concentric sclerosis. WM, white matter.

Fig. 2. T1 post-gadolinium injection sequences showing peripheral enhancement demonstrating an open ring shape aspect.

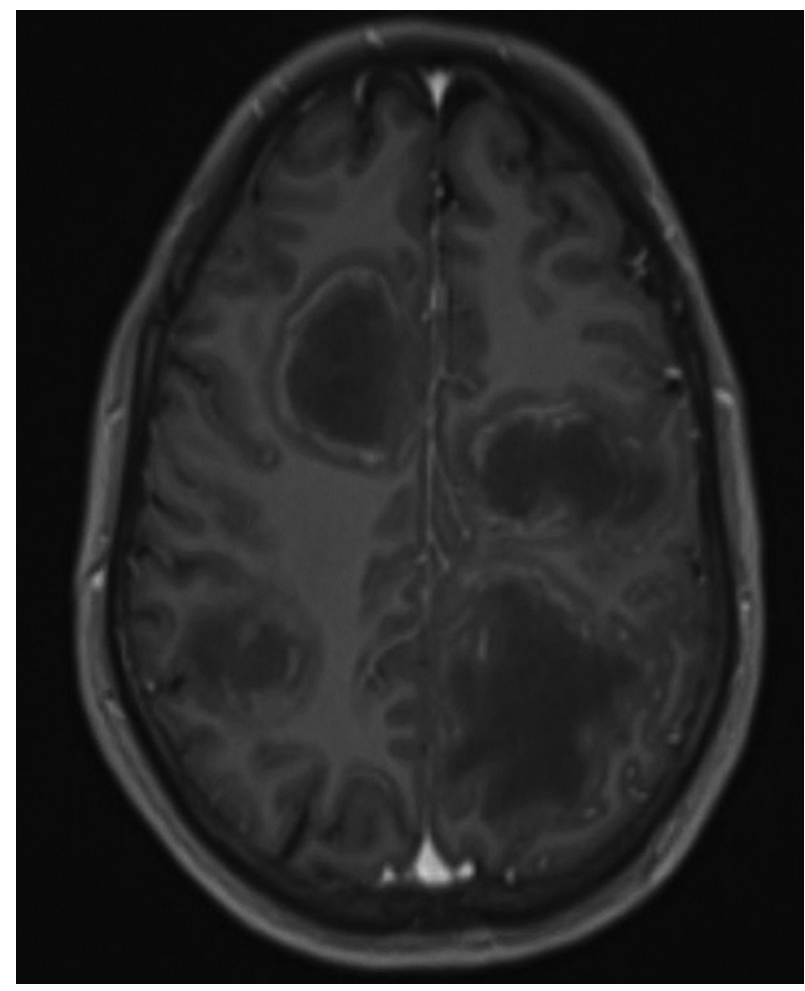

enhancement (shown in Fig. 2). An apparent diffusion coefficient image showed restricted diffusion at the periphery of the lesions (shown in Fig. 3).

Based on radiological findings consisting of the demyelinating nature of lesions, differential diagnoses such abscess and neoplastic processes were discarded. And owing to the 
Fig. 3. ADC map showing restricted diffusion at the periphery of the lesions. ADC, apparent diffusion coefficient.

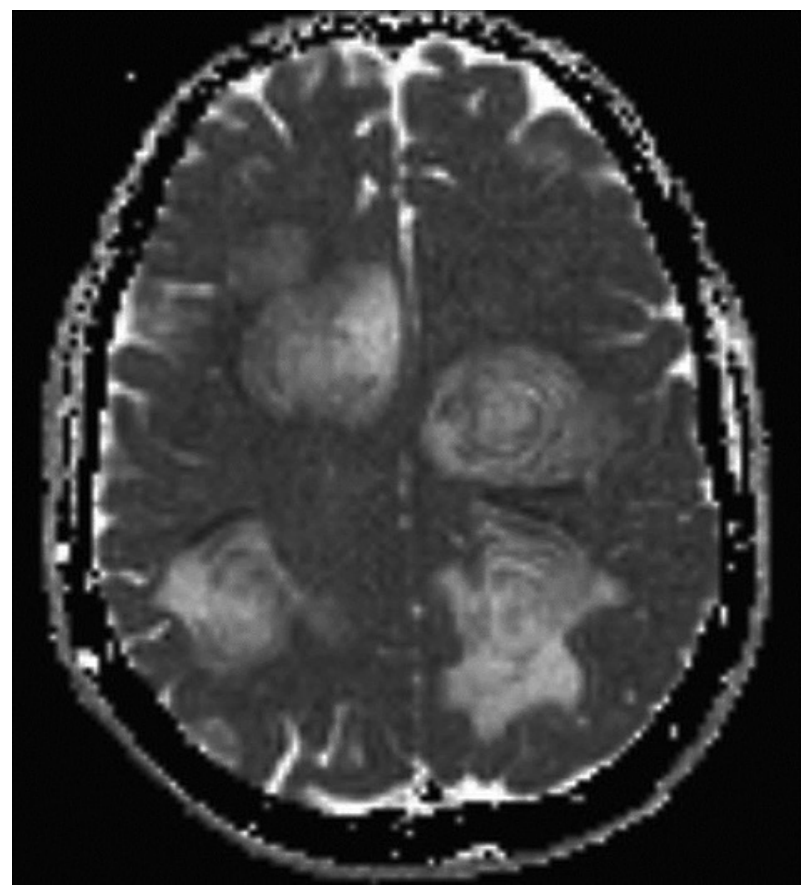

clinical presentation marked by an encephalopathy and the absence of any evidence of anterior dissemination of lesions in both space and time on clinical grounds and MRI findings, the diagnosis of multiple sclerosis was ruled out in favor of ADEM.

The patient was put on intravenous corticosteroids (methylprednisolone) at the dose of $1 \mathrm{~g}$ per day. Over the course of the following 10 days, he started regaining some of his neurological functionalities and has gone from an Expanded Disability Status Scale (EDSS) score of 6 to a score of 4 . Hereafter, the patient was discharged and put on oral prednisone with a starting dose of $1 \mathrm{mg} / \mathrm{kg} /$ day during the first week and tapered over the period of 6 weeks.

After 3 months, the patient recovered his speech ability, and his right lower and upper limb muscle strength went up to 4/5 on the MRC scale. His EDSS score went down to 1. On his brain MRI (shown in Fig. 4, 5), the pseudotumoral lesions have shrunk, with decreased diffusion on apparent diffusion coefficient images compared to the initial MRI. There was no longer an enhancement of lesions in post-gadolinium injection sequences.

\section{Discussion}

Pseudotumoral forms of demyelination are the expression of several demyelinating disease varieties, such as tumefactive demyelinating lesions, Balo's concentric sclerosis, Schilder's sclerosis, and Marburg disease. These overlapping presentations are not bounded to be only associated with multiple sclerosis but also could be seen in other demyelinating conditions, including ADEM, MOG-antibody disease (MOG-AD), and NMOSD [1].

Tumefactive demyelination indicates lesions $>2 \mathrm{~cm}$ in diameter; it manifests clinically with altered consciousness, seizures, and cognitive and polyfocal neurological deficits. Tumefactive demyelinating lesions could be single or multiple, with MRI findings suggestive of open ring enhancement, with minimum edema and mass effect contrasting with lesion size [3]. In CSF analysis, slightly elevated concentrations of proteins and mild pleocytosis are commonly found. Intrathecal oligoclonal bands are less common at the first clinical 

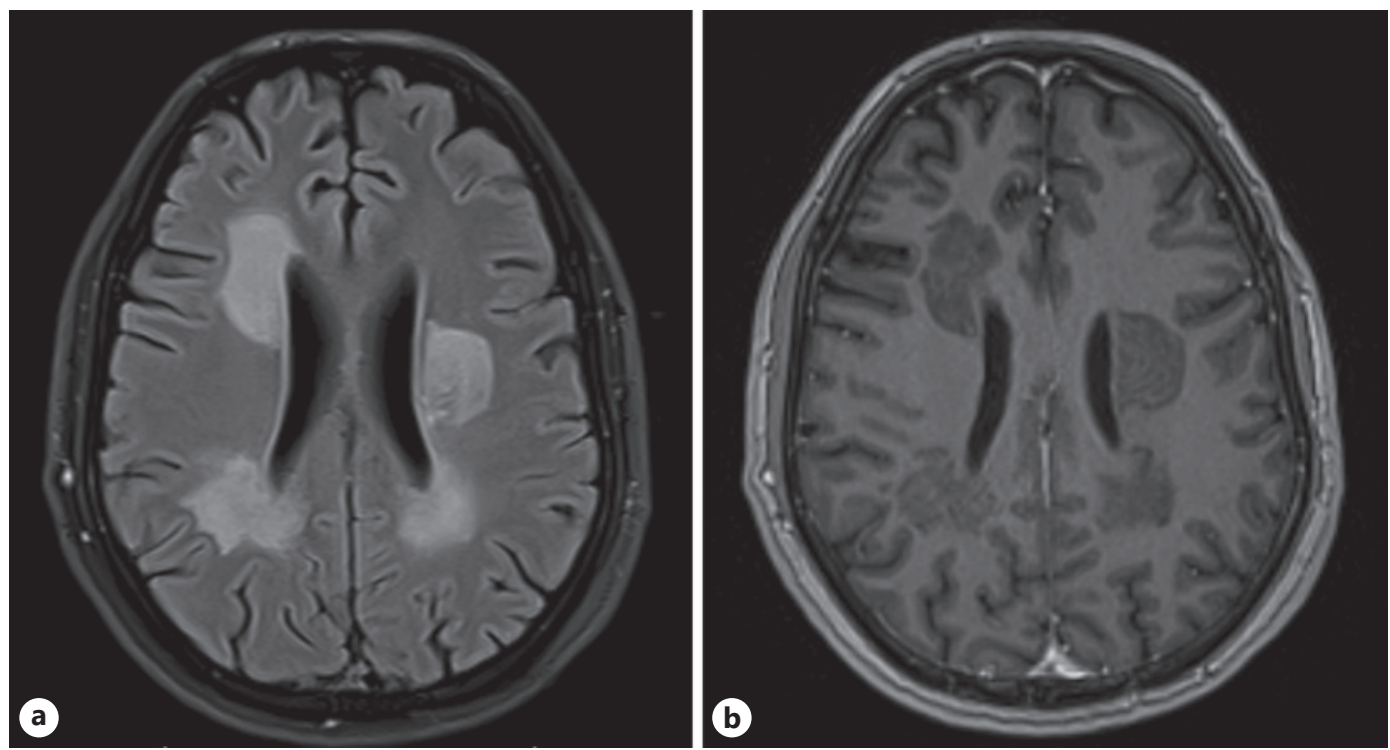

Fig. 4. Brain MRI after 3 months show a decrease in the lesion size on FLAIR sequence (a), and absence of enhancements in T1 post-gadolinium injection sequences (b).

Fig. 5. ADC image after 3 months showing a regression of diffusion in the lesions site. ADC, apparent diffusion coefficient.

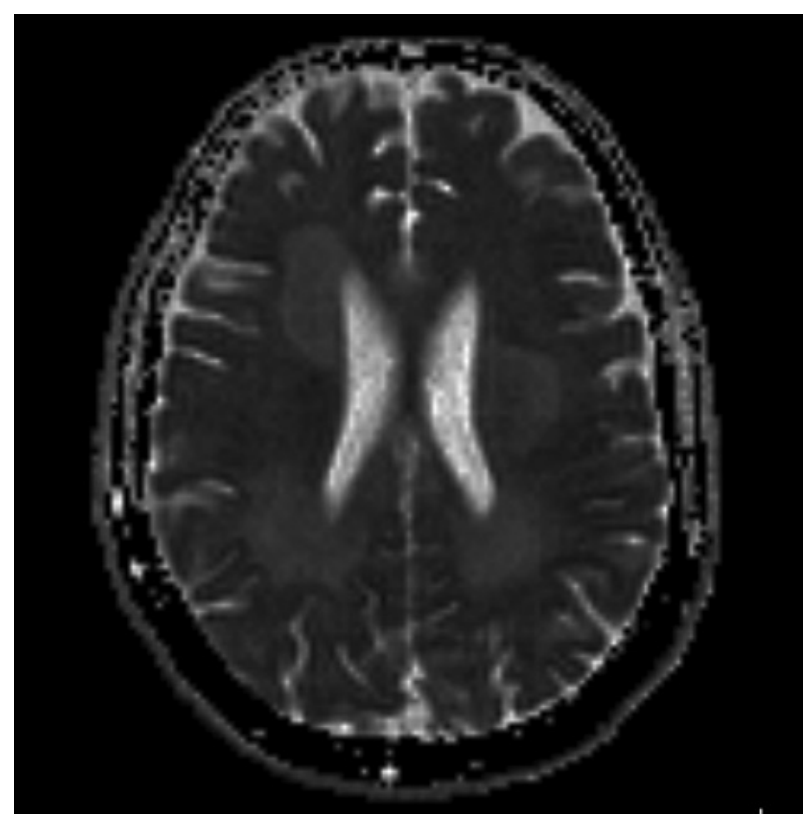

event than when lesions are found during the course of established diagnosis of MS (52 vs. 90\%) [4]. Prognosis and management of patients with tumefactive demyelinating lesions depend on the underlying demyelinating disease, with corticosteroids as the first-line treatment [5].

Balo's concentric sclerosis is a form of pseudotumoral demyelination in which lesions are multilayered in ring shape (onion-like image), with alternating areas of preserved myelin [5]. It is characterized by prodromal fever and headaches, and women predominance [6]. On MRI, lesions appear as concentric rings with alternating high and low intensities in T2-weighted and FLAIR sequences, with peripheral restriction in diffusion-weighted 
imaging [5]. CSF analysis is usually normal, and intrathecal oligoclonal bands are rarely seen compared with MS (35 vs. 98\%) [1]. Treatment consists of administering a high dose of corticosteroids or plasma exchange, or both, and immunosuppressive agents in nonresponding cases [6].

Neuromyelitis optica, also called Devic's disease, is a CNS inflammatory affection that differs from MS, usually associated with serum aquaporin-4 immunoglobulin $G$ antibodies (AQP4-IgG). Historically, its definition includes the presence of optic neuritis and longitudinally extensive transverse myelitis [5]. Currently, it is subsumed in what called NMOSD that requires patients having core clinical characteristics or related MRI findings referring to optic neuritis, acute myelitis, area postrema syndrome, acute brainstem syndrome, symptomatic narcolepsy, acute diencephalic clinical syndrome, or cerebral syndrome. In 2015, the diagnostic criteria for NMOSD with and without AQP4-IgG, or with the unknown AQP4-IgG status were established [7]. On MRI, lesions could be tumefactive with less discrete gadolinium enhancement than seen in MS [5]. On CSF analysis, restricted OCB synthesis is less frequently found than in MS ( $<20$ vs. $>80 \%$ ), with mononuclear pleocytosis $>50 / \mathrm{mm}^{3}$ [7]. NMOSD management is based on the corticosteroid administration, sometimes combined with or replaced by plasma exchange in refractory cases. For long-term treatment, consensus guidelines advocate oral corticosteroids with a concomitant immunosuppressive agent [5].

MOG-AD is considered as a distinct entity that differs from MS and NMOSD; it is a CNS inflammatory and demyelinating condition characterized by a monophasic or relapsing disease course. Its mean age of occurrence is early mid-30s with a subtle women predominance. The most frequent clinical manifestation is optic neuritis, usually bilateral, followed by myelitis, ADEM, or an ADEM-like presentation. Relapses (reported in 44-83\% of patients), when occurred, usually involve optic neuritis, yet with better visual field outcomes than in patients with AQP4-positive NMOSD [8]. CSF pleocytosis is variable; it is seen in up to 44-85\% of patients, with unusual intrathecal OCB synthesis. In order to reduce the chances of falsenegative results, MOG-IgG detection in serum should be carried using cell-based assay such as an indirect fluorescence test or fluorescence-activated cell sorting. MRI shows generally bilateral, ovoid, and poorly bordered lesions that appear as hypersignal images in T2-weighted and FLAIR sequences. The optic nerve is more edematous with extensive inflammatory lesions compared to AQP4-positive NMOSD patients [9]. In some cases, tumefactive demyelinating lesions are presented as the clinical and radiological manifestation in MOG-AD [1]. Management protocols are not so different from those adopted for treating NMOSD patients. Still, in the presence of poor prognostic factors for recurrence and/or disability, we resort to immunosuppressant agents [9].

ADEM, or postinfectious encephalomyelitis, is an immune-mediated inflammatory and demyelinating disease of the CNS which usually follows a nonspecific infection or a vaccination several weeks prior the first symptom onset. It is mostly seen in the pediatric population but can also occur in adults, with no gender predominance [10]. Clinically, neurological deficits are reflected by the location of demyelinating lesions, and as opposed to multiple sclerosis, encephalopathy is quite frequent in ADEM. Brain MRI findings typically include multiple large and asymmetrical hypersignal areas of demyelination in T2-weighted and FLAIR sequences, with poorly defined borders. Moreover, tumefactive demyelinating lesions can occur as well in ADEM [5]. Gadolinium enhancement is highly variable and believed to be probably depending on the time when MRI was performed over the disease course [11]. Lesions usually implicate the cortical and central WM, cortical gray-WM junction, thalami, basal ganglia, cerebellum, and brainstem [10]. CSF examination typically reveals a mild pleocytosis of $50 / \mathrm{mm}^{3}$, with or without high protein levels. Intrathecal OCB synthesis is usually absent. MOG-IgG antibodies are more frequently found in children than in adults [5]. ADEM first-line treatment consists of a high dose of intravenous corticosteroids. In case of contraindication

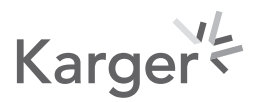


or ineffectiveness of the steroids, plasma exchange and intravenous immunoglobulin could also be used [10].

Assigning the diagnosis of ADEM to pseudotumoral demyelinating lesions is a delicate process as it is tricky to distinguish from an atypical presentation of multiple sclerosis. Normally, the presence of an encephalopathy that goes from behavioral changes, altered consciousness to coma is more suggestive of ADEM diagnosis [5], along with the absence of clinical and MRI proof of spatial and temporal dissemination of lesions.

As seen previously, diagnostic criteria are poorly defined, and the exact nosological classification remains inconclusive. Thus far, no clear consensus exists, which results in uncertainties and disagreements among authors. Some believe that pseudotumoral demyelinating lesions are confined only to an association with multiple sclerosis; others posit that they could also occur in other disease processes such in ADEM, MOG-AD, and NMOSD as it was clearly reported here in our case. The clinical and radiological findings were identical to those normally seen in ADEM patients.

\section{Conclusion}

Pseudotumoral demyelinating lesions are an uncommon presentation of demyelinating disorders. It is a rare entity that poses a major diagnostic challenge regarding the distinction between the different varieties and the recognition of the underlying disease. This diagnostic ordeal raises several questions in front of similar clinical presentations, especially in terms of prognosis, long-term evolution, the nomenclature of recurrent events, and basic treatment depending on the underlying demyelinating disease.

So, is it possible to be sure of a diagnosis based on 1 event or lesion type? Can we determine the risk of an eventual pseudotumoral demyelination presentation of ADEM to turn out to be multiple sclerosis? Could we consider each pseudotumoral demyelination to be an ADEM at the first encounter? Is it necessary to propose a relay by immunosuppressants immediately after the first episode, or do we ought to wait for a hypothetical second attack to conclude?

Greater clarity and uniformity are needed while talking about pseudotumoral demyelinating lesions. Hence, further studies are required to sort out this issue and to expand our knowledge about this challenging pathological condition with the ultimate goal of determining optimal courses of treatment.

\section{Statement of Ethics}

Written informed consent was given by the patient himself for publishing this case report and any accompanying images.

\section{Conflict of Interest Statement}

The authors declare that there is no conflict of interest regarding the publication of this manuscript.

\section{Funding Sources}

No funding was received to work on or publish this case report.

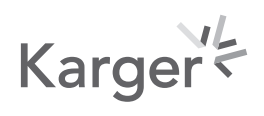




\section{Author Contributions}

Rachid Belfkih was the physician responsible for the patient and supervisor of the writing of this case report, Omar Ghomari Khayat and Hind H'daidane are the writers, and Fatima Zahra El Amrani did critical revisions of the content.

\section{References}

1 Hardy TA. Pseudotumoral demyelinating lesions: diagnostic approach and long-term outcome. Curr Opin Neurol. 2019 Jun;32(3):467-74.

2 Comi G. Multiple sclerosis: pseudotumoral forms. Neurol Sci. 2004 Nov;25(Suppl 4):S374-9.

3 Kiriyama T, Kataoka H, Taoka T, Tonomura Y, Terashima M, Morikawa M, et al. Characteristic neuroimaging in patients with tumefactive demyelinating lesions exceeding $30 \mathrm{~mm}$. J Neuroimaging. 2011;21:e69-77.

4 Altintas A, Petek B, Isik N, Terzi M, Bolukbasi F, Tavsanli M, et al. Clinical and radiological characteristics of tumefactive demyelinating lesions: follow-up study. Mult Scler. 2012;18:1448-53.

5 Hardy TA, Reddel SW, Barnett MH, Palace J, Lucchinetti CF, Weinshenker BG. Atypical inflammatory demyelinating syndromes of the CNS. Lancet Neurol. 2016 Aug;15(9):967-81.

6 Hardy TA, Miller DH. Baló's concentric sclerosis. Lancet Neurol. 2014;13:740-6.

7 Wingerchuk DM, Banwell B, Bennett JL, Cabre P, Carroll W, Chitnis T, et al. International consensus diagnostic criteria for neuromyelitis optica spectrum disorders. Neurology. 2015;85:177-89.

8 Mariotto S, Ferrari S, Monaco S, Benedetti MD, Schanda K, Alberti D, et al. Clinical spectrum and IgG subclass analysis of anti-myelin oligodendrocyte glycoprotein antibody-associated syndromes: a multicenter study. J Neurol. 2017 Dec;264(12):2420-30.

9 Wynford-Thomas R, Jacob A, Tomassini V. Neurological update: MOG antibody disease. J Neurol. 2019;266(5): 1280-6.

10 Filippi M, Rocca MA. Acute disseminated encephalomyelitis. White Matter Dis. 2020 Feb 12;109-25.

11 Berzero G, Cortese A, Ravaglia S, Marchioni E. Diagnosis and therapy of acute disseminated encephalomyelitis and its variants. Expert Rev Neurother. 2016;16(1):83-101. 